\title{
The Impact of Subway on Urban Traffic Network Based on Complex Network Theory
}

\author{
Cao Hongmei ${ }^{a}$, Zhao Fang, Liu Hao and Yu Tong \\ Inner Mongolia University, China
}

\begin{abstract}
In order to explore changes in accessibility and connectivity of urban traffic network before and after the construction of subway, traffic networks within the Second Ring road, Hohhot, was analyzed based on complex network theory. Firstly, the urban road traffic network and the urban compound traffic network with the adjunction of the subway to former were mapped. Secondly, the node degree, clustering coefficient, average path length and node strength of the two networks were calculated based on SuperMap. Finally, the eigenvalues obtained above were compared and analyzed. Results show that both of the two traffic networks have some characteristics of small clustering coefficient reflected in random network and scale-free network. The latter, compared to the former, network diameter and average shortest path reduce, the average node degree and average node intensity increase. That is to say, with the adjunction of the subway, the connectivity and the accessibility of the entire network are significantly improved, and its carrying capacity increases remarkably.
\end{abstract}

\section{Introduction}

Subway plays an important role in improving traffic conditions as a high capacity and long distance and fast transportation mode, and with the adjunction of subway, the structure and evolution of urban traffic network will be impacted. Because topological structure of urban traffic system are similar to that of complex networks, many scholars make traffic networks researches based on complex network theory. Some studies on urban compound traffic network which is a combination of subway network and road network are conducted respectively in Statistical topological Characteristics, carrying capacity and evolution [1-3]. Based on complex network theory, in this paper, the impact of subway on character, structure and carrying capacity of the urban traffic network were concluded by comparing and analysing its statistics eigenvalues before and after the construction of subway. It will help to improve the performance of urban traffic network in management practice, and provide guidelines for transportation planning in future.

\section{Urban traffic network constructions}

In order to explore changes in accessibility and connectivity of urban traffic network before and after the construction of subway, Urban Road Traffic Network and Urban Compound Traffic Network were constructed. For research convenience, the former only considered the expressways, primary arterials and secondary arterials within the Second Ring road, Hohhot. The latter

\footnotetext{
${ }^{\mathrm{a}}$ Corresponding author: Cao Hongmei, xghcao@163.com
}

contained subway based on the former. In addition, it's assumed that both of them were undirected networks.

\subsection{Urban road traffic network}

With links and nodes representing road segments and junctions, the urban road network is built. Geert man and van Eck claims that this option is attractive as many existing modelling programmes can be used without much change [4]. By use of GIS integrated graphics and attribute data management capabilities, edit and update road network information, combined with the characteristics of urban road traffic road network of Hohhot weighted network dataset is added as a GIS layer. The network consists of 169 nodes, 290 edges, as shown in Figure 1.

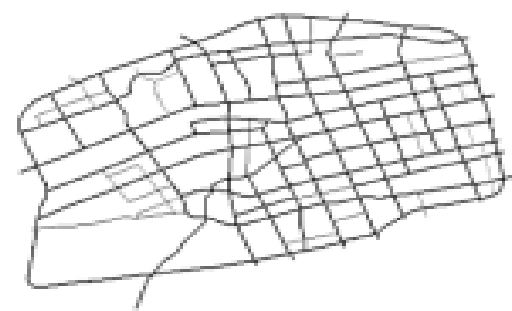

Figure 1. Hohhot road traffic network

\subsection{Urban compound traffic network}

With the subway stations as nodes, the lines connecting sites as sides, the urban compound traffic network with 
the adjunction of urban subway was constructed. The urban compound traffic network consists of 172 nodes, 294 edges, as shown in Figure 2.

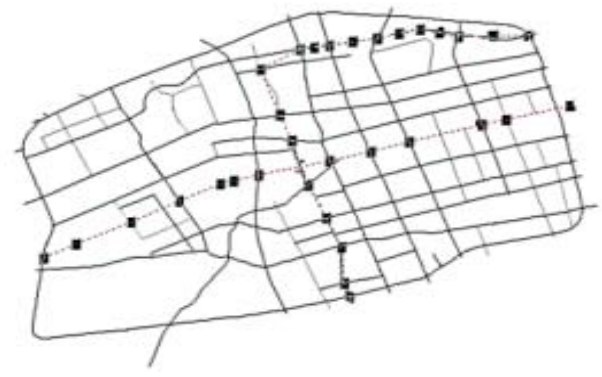

Figure 2. Hohhot compound traffic network

\section{Calculation and comparative analysis of eigenvalues}

\subsection{Comparison and analysis of the degree and degree distribution function}

Degree is defined as the degree of node $i$, it represents the number of edges connected to node $i$, the greater is, the greater the influence node $\mathrm{i}$ has on the entire network; represents the degree distribution function, which refers to the probability that a node has a number of $\mathrm{k}$ edges connected in network, and it reflects the macro-statistical characteristics of the network system. The distribution is in line with Poisson distribution indicating that the network has the characteristics of small-world network, in line with the distribution of power indicating that the network has a scale-free property.

Table 1. Node degree Characteristics of complex network models of different topologies

\begin{tabular}{|c|c|c|c|c|c|c|}
\hline \multirow[t]{2}{*}{ Degree } & \multicolumn{3}{|c|}{ Road traffic network } & \multicolumn{3}{|c|}{ Compound traffic network } \\
\hline & $\begin{array}{c}\text { Number } \\
\text { of } \\
\text { nodes }\end{array}$ & Percentage & Average & $\begin{array}{c}\text { Number } \\
\text { of } \\
\text { nodes }\end{array}$ & Percentage & Average \\
\hline 1 & 2 & 0.012 & \multirow[t]{8}{*}{3.592} & 2 & 0.012 & \multirow[t]{8}{*}{3.890} \\
\hline 2 & 5 & 0.030 & & 5 & 0.029 & \\
\hline 3 & 53 & 0.314 & & 50 & 0.291 & \\
\hline 4 & 109 & 0.645 & & 91 & 0.529 & \\
\hline 5 & 0 & 0 & & 3 & 0.017 & \\
\hline 6 & 0 & 0 & & 20 & 0.116 & \\
\hline 7 & 0 & 0 & & 0 & 0 & \\
\hline 8 & 0 & 0 & & 1 & 0.006 & \\
\hline
\end{tabular}

Table 1 showed us that both networks haven't presented strictly Poisson or exponential probability distribution.

\subsection{Comparison and analysis of diameter and average path lengths}

Distance between two nodes $i$ and $j$ in network is defined as the number of edges of the shortest path connecting the two nodes. The average path length A refers to the mean shortest path length between any two nodes, which can be formulated as:

$$
A=\frac{1}{\frac{N(N+1)}{2}} \sum_{\mathrm{i} \geq j} \mathrm{~d}_{\mathrm{ij}}
$$

where: $\mathrm{N}=$ the number of nodes in network

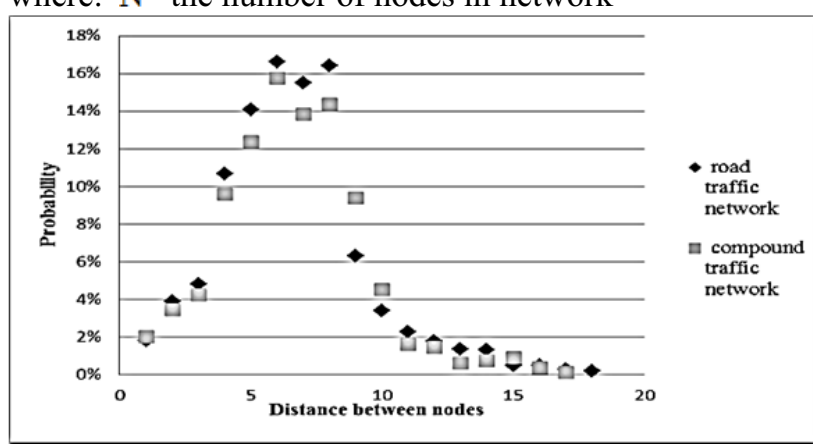

Figure 3. Distribution of distance between nodes in unban traffic network

The diameter and average path lengths of the network can be calculated based network analysis tools on Supermap Deskpro. The results show that Diameter of the urban compound traffic network is 17 , the average shortest path length is 6.2731 instead of 18 and 6.9036 in the urban road traffic network respectively. These decreases reflect that fewer numbers of sections are needed for nodes to reach each other. The distributions of distance between nodes in two networks are shown at Figure 3. It shows that the dispersion degree about distribution of shortest path between nodes in compound traffic network reduced, the probability in the central region $[5,10]$ become larger, and the tightness of the network nodes increase.

\subsection{Comparison and analysis of clustering coefficient}

Clustering coefficient of a node refers to the proportion of the number of edges between its neighboring nodes and the largest possible number of connected edges, which can be formulated as:

$$
C_{i}=\frac{2 E_{i}}{k_{i}\left(k_{i}-1\right)}
$$

where: $\mathrm{E}_{\mathrm{i}}=$ the number of edges between node $i$ and its neighboring nodes.

The clustering coefficient $\mathrm{C}$ of entire network is average of all clustering coefficient $C_{i}$ of node $i$, where, $0 \leq \mathrm{C} \leq 1 . \mathrm{C}=0$ means that all nodes are isolated nodes; $\mathrm{C}$ $=1$ means the network is a global coupled, in other words, any two nodes in network are directly connected. Because clustering coefficient the network may show a characteristic of random nature or small world [6].

Table 2. Clustering coefficient characteristics of complex networks

\begin{tabular}{|c|c|c|c|c|}
\hline \multirow{2}{*}{$\begin{array}{c}\text { Clustering } \\
\text { coefficient }\end{array}$} & \multicolumn{2}{|c|}{$\begin{array}{c}\text { Road traffic } \\
\text { network }\end{array}$} & \multicolumn{2}{|c|}{$\begin{array}{c}\text { Compound traffic } \\
\text { network }\end{array}$} \\
\cline { 2 - 2 } & Percentage & Average & Percentage & Average \\
\hline 0 & $91.12 \%$ & 0.01874 & $93.02 \%$ & 0.01395 \\
\cline { 2 - 2 } & 0 & & $0.58 \%$ & \\
\cline { 2 - 2 } & & & $4.65 \%$ & \\
\hline $1 / 15$ & $2.51 \%$ & & $1.75 \%$ & \\
\hline $1 / 3$ & $2.37 \%$ & & \\
\hline
\end{tabular}

The average clustering coefficient of road traffic network and compound traffic network are 0.01874 and 0.01395 respectively. They are less than 1 to a large 
extent which shows that they all don't have small world characteristic. Table 2 presents a comparison of clustering coefficient characteristics between road traffic network and compound traffic network. The proportion of nodes that have 0 clustering coefficient reaches to more than $90 \%$, showing the lack of overall network partial connectivity and less intensive

\subsection{Comparison of the intensity of node}

Edge intensity is a measure of the importance of edges in the network. Generally, different types of edge have different intensity value as shown Table 3 . Intensity of a node is the sum of all connected edge intensity. Edge intensity and node intensity limit the capacity of the network.

Table 3. Edge intensity

\begin{tabular}{|l|l|l|l|l|}
\hline Types of edge & Subway & Expressway & $\begin{array}{l}\text { Main } \\
\text { road }\end{array}$ & $\begin{array}{l}\text { Secondary } \\
\text { road }\end{array}$ \\
\hline $\begin{array}{l}\text { Traffic capacity(Million } \\
\text { passengers / hour) }\end{array}$ & 4.400 & 1.344 & 1.176 & 0.672 \\
\hline Edge intensity & 1 & 0.31 & 0.27 & 0.15 \\
\hline
\end{tabular}

Table 4. Node intensity

\begin{tabular}{|l|l|l|l|}
\hline Node intensity & Maximum & Minimum & Mean \\
\hline Road traffic network & 1.24 & 0.15 & 0.9251 \\
\hline Compound traffic network & 5.08 & 0.15 & 1.2440 \\
\hline
\end{tabular}

Maximum, minimum and mean of node intensity are showed in Table 4 . The intensity of node in network that connects to subway lane is obviously higher than others. Statistics obtained that $56 \%$ of the subway stations are connected to the intersection of two trunks to achieve superimposed.

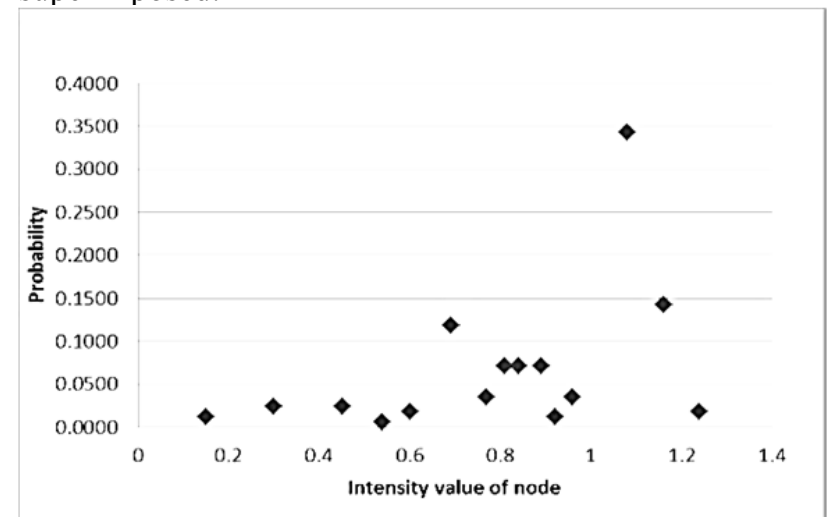

Figure 4. Distribution of node intensity inroad traffic network

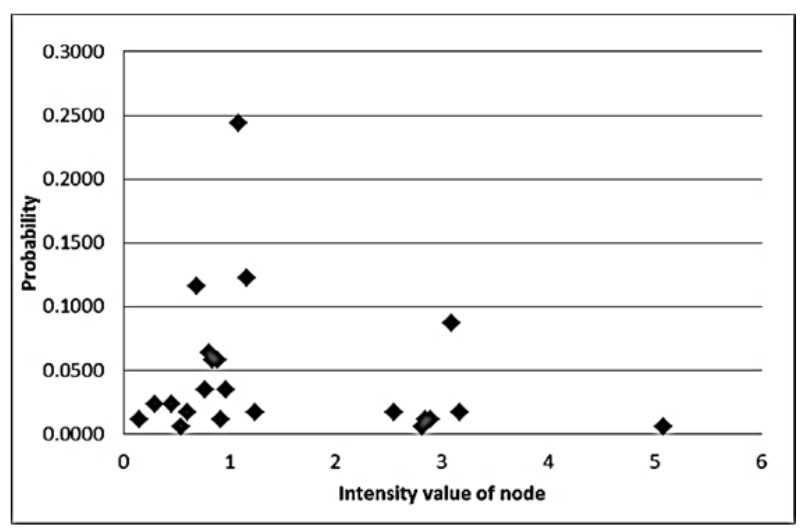

Figure 5. Distribution of node intensity in compound traffic network

\section{Summary}

Compare and analysis the overall network performance as is shown in Table 5, diameter and average path lengths in compound traffic network reduce by $5.6 \%$ and $0.9 \%$ respectively, compared to road traffic network, reflecting the connectivity of network has significantly improved, the amount of traffic carried by network increases, residents can choose more routes than before, the distance and time required in a travel become shorter, the accessibility of the entire network is improved remarkably. In addition, as it presented in Table 4, statistical eigenvalues of key nodes in network change a lot, which makes the traffic distribution becomes more balanced, more stable the network traffic is, especially the junction of two subway lines, Xinhua Square Station, the degree and intensity of it increase by $100 \%$ and $370 \%$ respectively, indicating its influence in the whole network has been greatly improved, to some extent, it shares the traffic pressure of Hailiang Plaza, however, strengthen of guidance and supervision also are necessary.

Table 5. Statistical indicators

\begin{tabular}{|l|l|l|}
\hline Statistical indicators & $\begin{array}{l}\text { Road traffic } \\
\text { network }\end{array}$ & \multicolumn{1}{|c|}{$\begin{array}{c}\text { Compound traffic } \\
\text { network }\end{array}$} \\
\hline Average degree & 3.592 & 3.890 \\
\hline Average path lengths & 6.9036 & 6.2713 \\
\hline Network diameter & 18 & 17 \\
\hline $\begin{array}{l}\text { Average clustering } \\
\text { coefficient }\end{array}$ & 0.01874 & 0.01395 \\
\hline Average node intensity & 0.9251 & 1.2440 \\
\hline
\end{tabular}

\section{References}

1. ZL. Wei, YJ. Gan and P. Zhao. Characteristic Research of Urban Complex Traffic Network [ J ] .Journal of Transportation Systems Engineering and Information Technology. 15 ( 2015)

2. P. Zhao. Collaborative Study on Multi-Modal Urban Traffic Network Based on Complex Network Theory-Urban Road Traffic Network and Urban Rail Transit Network [ D ] .Beijing :Beijing jiaotong university(2014) 
3. W. Liu. Research on the effect of mass transit on complex network theory[D]. Harbin Institute of Technology(2009)

4. Geertman and Ritsema van Eck. GIS and models of accessibility potential: an application in planning. Int. J. Geographical Information Systems, 9, 67-80(1995)

5. XF. Wang, X. Li and GR. Chen. complex networks and applications Edited [M] Beijing: Tsinghua University Press(2006)

6. Watts D J, Strogatz S H. Collective dynamics of 'small-world' networks [J]. Nature, 393: 440-442. (1998)

7. Albert-László, Barabási, Erzsébet Ravasz, et al.Deterministic Scale-Free Networks[J]. Physica A: Statistical Mechanics and its Applications, 29963(34): 559-564(2001)

8. Albert R, Barabasi A L. Statistical mechanics of complex networks [J]. Reviews of ModernPhysics. 74: 47-97(2002) network on the urban transportation network based

9. H. Corley and D. Y. Sha, "Most vital links and nodes in weighted networks," Operations Research Letters, 1, 157-160(1982)

10. Albert-L'aszl'o Barab'asia, Erzs'ebet Ravasza, Tam'as Vicsekb Deterministic Scale-Free Networks(2008)

11. KM. Chen and BJ. Yan. Analyze of the road capacity, Beijing: People's Communication Press(2003)

12. Dalvi M. Q. and Martin K. M. The measurement of accessibility: Some preliminary results. Transportation, 5, 17-42 (1976)

13. M Barthélemy. Crossover from Scale-free to Spatial Networks [J]. Europhys. Lett., 63(6),915-921(2003)

14. YM. Lu and JM. Tang. Fractal Dimension of a Transportation Network and its Relationship with Urban Growth: a study of the Dallas - Fort Wortharea $[\mathrm{J}]$. Environment and Planning B: Planning and Design, 31,895-911(2004) 